\title{
Integrating Medical Images into Hospital Information Systems
}

\author{
Ruth E. Dayhoff, Daniel L. Maloney, Peter M. Kuzmak, and Barclay M. Shepard
}

\begin{abstract}
The effective delivery of health care has become increasingly dependent on a wide range of medical data that includes a variety of images. Manual and computer-based medical records ordinarily do not contain image data, leaving the physician to deal with a fragmented patient record widely scattered throughout the hospital. The Department of Veterans Affairs is currently installing a prototype hospital information system workstation network to demonstrate the feasibility of providing image management and communications functionality as an integral part of an existing hospital information system. The core of this system is a data base management system adapted to handle images as a new data type. A general model for this network-based integration is discussed and various alternative architectures are examined.

This is a US government work. There are no restrictions on its use.
\end{abstract}

KEY WORDS: image management, hospital information system, HIS, image integration, data type, multimedia data base management system, automated clinical record.

$\mathbf{T}$ ODAY, health care is being delivered more and more by institution rather than by individual physician. ${ }^{1}$ Many consulting physicians collect data, often image data, that is stored in the consulting department. Once an exam is complete, the treating physician may have difficulty gaining access to the images because only one copy is available in the hospital, and it is being used or has been misplaced. Because medical decisions must often be made quickly, the treating physician may end up choosing a treatment strategy based on the advice of the consulting physicians (who were not provided with comprehensive patient data) without seeing the image data on which that advice was based. Clinicians need timely access to both patient's medical images and the alphanumeric data typically provided by hospital information systems. An automated system that combines the patient's image and text data will provide the treating physician with a more complete view of the patient's data.

There are several major needs that must be met by the comprehensive hospital information system of the future. The first is to provide the patient's complete medical data to the treating physician, throughout the patient's stay. The second need is to provide the patient's complete data to the consulting physicians in other depart- ments, both to aid in the consultation process and to provide feedback about patient diagnosis and outcome. The comprehensive hospital information system (HIS) should provide this multiway communication among physicians that has been difficult in the past.

Another requirement of future HIS's is that they present the patient's data in an integrated fashion, whether it is image data, alphanumeric data, EKG data, or other data types. That is, a system should maintain the correct relationships between the various data collected regarding a patient so that its presentation supports medical decision making. This task may require a controlling system that maintains the data model and references to the patient's data. There is conceptually a single data dictionary, but with today's advances in distributed systems, its components may reside in different locations. $^{2}$

Finally, it is important that the system provide a simple and consistent access procedure to the patient's data. This can be done initially by utilizing a familiar user interface. However, as new data types are added to the system, there are new challenges in developing an appropriate user interface.

Medical images of importance include images resulting from cardiology studies, microscopic pathology slide views, radiology studies, dermatologic or ophthalmologic examinations, nuclear medicine scans, and endoscopic exams. A number of medical devices produce digitized images for use in medical diagnosis and treatment. Typically, these are standalone devices, and image data is stored either in analog form on films or digitally on magnetic media. Recently, researchers and manufacturers have begun developing standalone image management and communications systems (IMACS) capable

From the Washington Information Systems Center, Department of Veterans Affairs, Washington, DC.

Address reprint requests to Ruth E. Dayhoff, $M D$, Washington Information Systems Center (10B/ISC2), 8403 Colesville Rd, Suite 200, Silver Spring, MD 20910.

This is a US government work. There are no restrictions on its use.

0897-1889/91/0402-0001\$03.00/0 
of storing large numbers of patient images. However, the initial designs of these systems did not include communication with existing hospital information systems or departmental information systems (DIS). Instead the IMACS and the HIS or DIS were treated as standalone devices, rather than as part of a larger integrated communication system.

The radiological community has had a lot of experience with standalone IMACS. They have found that availability of radiologic images results in a decrease in time required to initiate patient treatment. ${ }^{3}$ However, these IMAC systems do not meet the need to provide integrated patient data to treating physicians. Levine et $\mathrm{al}^{4}$ noted that interfaces between radiology information systems and IMACS are necessary if IMACS are to become clinically accepted. Lodder et $\mathrm{al}^{5}$ noted that an HIS-picture archiving communication system (PACS) coupling was needed to supply the user with all relevant patient data at a single workstation. Most interfacing attempts to date have involved a "one way" passing of patient demographic and ordering data from the HIS or radiology information system (RIS) to the image system. ${ }^{4-6}$ The American College of Radiology-National Electrical Manufacturers Association standards committee has recently established a working group to examine more complete interfacing of IMACS to RIS and HIS systems. ${ }^{7}$ Several groups have done theoretical work developing a model for the overall entity relationships involved in such an interface. ${ }^{5,8}$ However, this work has generally involved interfaces between systems rather than true integration of the systems.

\section{DIFFICULTIES IN INTEGRATING IMAGE DATA WITH HOSPITAL INFORMATION SYSTEMS}

There are some inherent difficulties in combining image and text data in the same data management system. Image data is comprised of very large aggregates and requires rapid processing to be practical. Text data on the other hand comes in many small units and requires more complex data management. Typically, computer languages have been optimized for one task or the other. Additionally, large image size currently requires that images be stored on optical media for at least some portion of their life cycle. The handling of both types of data by the same system requires multiple technologies and a more complicated architecture than previously needed.

Another set of difficulties stems from the variety of hospital information system architectures. There are integrated hospital information systems where the needs of multiple departments are served by the same computer system, possibly in a distributed environment. There are also standalone departmental systems that meet the needs of an individual department and do not communicate with other systems. Finally, some hospitals have attempted to integrate their standalone departmental systems in a networked environment, but major problems are often encountered with patient identification discrepancies between systems, differences in knowledge representation between systems, synchronization of redundant data, and physical communication mismatches. Several groups are working on standards for medical data exchange ${ }^{9}$ and medical system integration, ${ }^{10}$ but few networks have as yet been fully implemented.

Integrated hospital information systems provide a simpler basis for integration of IMACS functionality because of their existing centralized data base management system and the homogeneity of the software. The Department of Veterans Affairs (VA) has such an integrated hospital information system, and is using it as the basis for image integration. The VA is undertaking a project to add IMACS functionality to this existing integrated system, recognizing that the benefits will be shared by its large installed base of systems. Details of this project have been described elsewhere. ${ }^{16,17}$

\section{CONCEPTUAL MODEL FOR SOFTWARE INTEGRATION}

One of the most important breakthroughs provided by data base systems is that they treat data in a general way. That is, the user can create a data base dictionary describing a data model, and use the same data base system to store information regardless of the particular data values or data types. The data base management system used in building the VA's hospital information system handles the following field types: free text, numeric data, dates/times, selections from short lists (data by reference), point- 
ers to lists (data by reference), word processing fields, and computed data. For each field in the data base dictionary, the developer has the option to perform additional data entry format checks and can include program code to perform unusual functions.

It is possible to extend this data base system to handle additional types of multimedia data such as: individual images, image sequences, text overlays, three dimensional reconstructions, audio data, or EKG data. It is important that such a multimedia data base system handle its various kinds of data in a general way that is transparent to the user. The new concept of object-oriented programming surrounds each data type with the software, or methods, required to handle it. ${ }^{11}$ To such a system, images, voice, and text are different data types that are handled by the same data base management software.

In providing IMACS functionality in an HIS, it is important to plan for a networked environment because there may be a need to locate different types of data on different kinds of data servers. A critical element in such a network is the presence of a system that maintains the data dictionary and relationship model for the data contained in the entire system. This is conceptually a single data dictionary, but an advanced distributed system may handle its actual location transparently. The presence of this overall relationship model allows the system to present the patient's data in an integrated fashion, whether it is image data, alphanumeric data, EKG data, etc. The system is automatically directed to the appropriate software modules in the network environment to obtain the data it needs. This capability has been called an "image broker" by some and a "redirector" by others. ${ }^{2}$ It can be used to support file migration functions. A second critical element is that the system contain a unique identification for each patient and that this same identification be used by all connected systems.

Given this conceptual software model, further system design choices are based on the existing hospital equipment and performance requirements. Various options exist for storage devices and data migration, network software, and the individual use of data by value or reference. Certain performance requirements must be met related to data display time, record searching and the creation of indices.

\section{POSSIBLE INTEGRATION ARCHITECTURES}

Three currently used image system interface/ integration architectures are shown in Figs 1,2, and 3 . These architectures have been driven by existing system components. These do not all match the design model outlined above, but will be discussed with respect to it.

\section{Model 1: No Interface or Integration}

The simplest model provides no communication between the HIS and IMACS. Separate terminals or windows on a workstation may be used for system access. In the latter case, using workstation software, the user opens a window to the HIS and inquires about patient text data. The user then opens a second window to the IMACS and inquires about the same patient's image data. The user must manually wrestle with discrepancies in patient identification. Unfortunately, with this architecture the user must enter patient data repetitively. A more sophisticated implementation would have the workstation software accept the patient identification data from the user once, and use it to request data from both systems. In this architecture, the IMACS performs image management and storage, while the HIS handles patient text data. There are separate data dictionaries and possibly discrepant patient identification information.

\section{Model 2: IMACS/HIS Interface}

This architecture keeps the HIS and IMAC system relatively separate but with some inter-

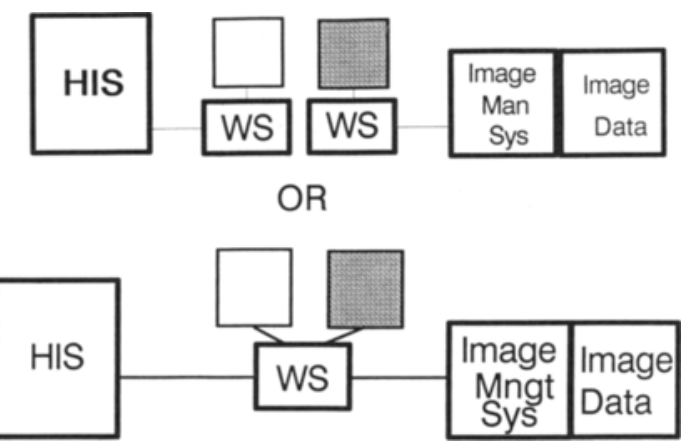

Fig 1. No interface or integration between IMACS and HIS or DIS (Model 1). 


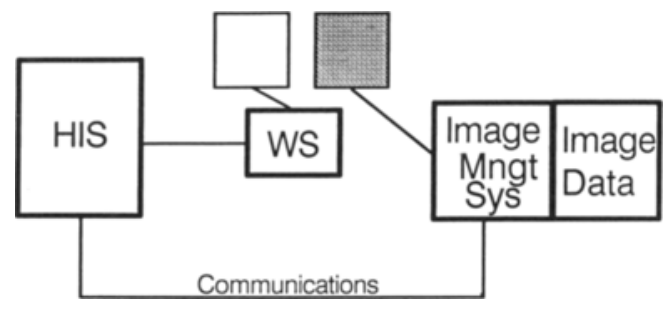

Fig 2. Interface between IMACS and HIS or DIS (Model 2).

communication. One or two-way communication exists between the HIS and the IMACS. Typically, the HIS sends patient identification information, and possibly, ordering information to the IMACS. The IMACS may provide confirmation of image acquisitions. The IMACS then automatically downloads the images to the workstation for viewing at a later time. The physician views the images on the IMACS workstation and prepares a consultation report which is then entered into the HIS. Both systems could use the same workstation, but this is not typical.

In this model, some patient data must be stored redundantly on both systems. There is generally no single comprehensive data dictionary on the system, instead it depends on "lookups" in each system. There is no record in the HIS of individual images stored on the IMACS. Rather the HIS has patient ID information and records of studies ordered. It relies on the IMACS to manage image retrieval based on patient ID. The user at the HIS workstation requests information from the HIS that in turn requests the IMACS to display a patient's image information on the image screen. This can be done in real time or as preloading of images based on studies that were recently performed. In this model, the IMACS performs image management and storage.

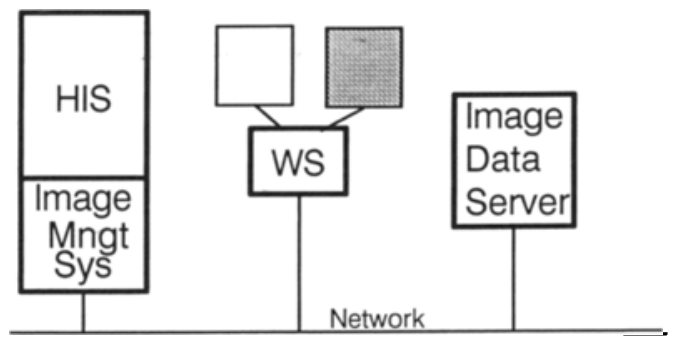

Fig 3. Integration at the HIS (Model 3).

\section{Model 3: Integration at the HIS}

This last model, used by the VA, moves the image management function from the IMACS to the HIS. In the VA's Decentralized Hospital Computer Program (DHCP) Integrated Imaging System, the workstation is running HIS software directly, obtaining patient specific text data across the network from the HIS. The complete data dictionary and image data references stored in the HIS allow the workstation to obtain images directly and display them in relationship to other patient data. Data produced by different medical specialties can be integrated in this way. Image data may reside on one or more image data servers connected to the network accessible to the workstation.

Models 1 and 2 depend on the two systems maintaining synchronized patient identification information. In model 3, the patient identification information is only stored once, in the integrated HIS.

We selected model 3 as our approach for a number of reasons. We felt that it allowed a conceptual model that provided the closest integration of the desired functionality. It allows a relatively simple and consistent user interface, and could be done efficiently with Massachusetts General Hospital Utility MultiProgramming System (MUMPS). We could maintain the VA's goals of an integrated system, and programmers will not need to be trained to maintain substantially different software.

\section{VA'S INTEGRATED HOSPITAL INFORMATION SYSTEM}

The VA's hospital information system, DHCP, is an integrated system that is installed in 164 VA medical centers. ${ }^{13,14}$ It was designed and implemented using an iterative rapid prototyping process involving both VA users and software developers that has been referred to as the "VA structure model" of medical software development. ${ }^{12}$ DHCP derivative systems are currently in use in virtually all VA Medical Centers, as well as the Department of Defense, the Indian Health Service, other federal and state agencies, and private hospitals in the United States and worldwide. ${ }^{13,14}$

The VA's DHCP system is different from 
multi-vendor systems in that it is an integrated system (see Fig 4) based on a central set of software development tools produced by the VA and available in the public domain. These tools include the VA File Manager, a powerful data base management system (DBMS) that also provides an extensive software development toolkit. Another set of tools, the VA Kernel, isolates all vendor dependent portions of the software in a small distinct table. Tools are also provided for menu management, security, task scheduling, and electronic mail.

All of the DHCP hospital information system is built using common programmer productivity tools. Because of programming conventions to store all data in the DBMS, both data and data dictionaries are available to all applications modules running across the multiple minicomputers that support the DHCP system (see Fig 4 , second ring from center).

By design, all data is stored in the DBMS, and both data and data dictionaries are available to all application modules running across the multiple minicomputers that support the DHCP system. The current VA DHCP software includes core modules for admission-dischargetransfer, scheduling, pharmacy, clinical laboratory, radiology, surgery, nursing, dietetics, records tracking, and a hospital wide ordering/ purchasing/fiscal package. In addition, applications have been added for medical specialty use (see Fig 4, third ring from center). The system's modular design, vendor independence, and emphasis on adherence to standards allows easy addition of new applications, and adaptation to new hardware developments.

Finally, there is another software layer that integrates data across modules for clinician interaction and results reporting. This includes an "order entry result reporting" module and a "patient health care summary" module (see Fig 4 , outer ring).

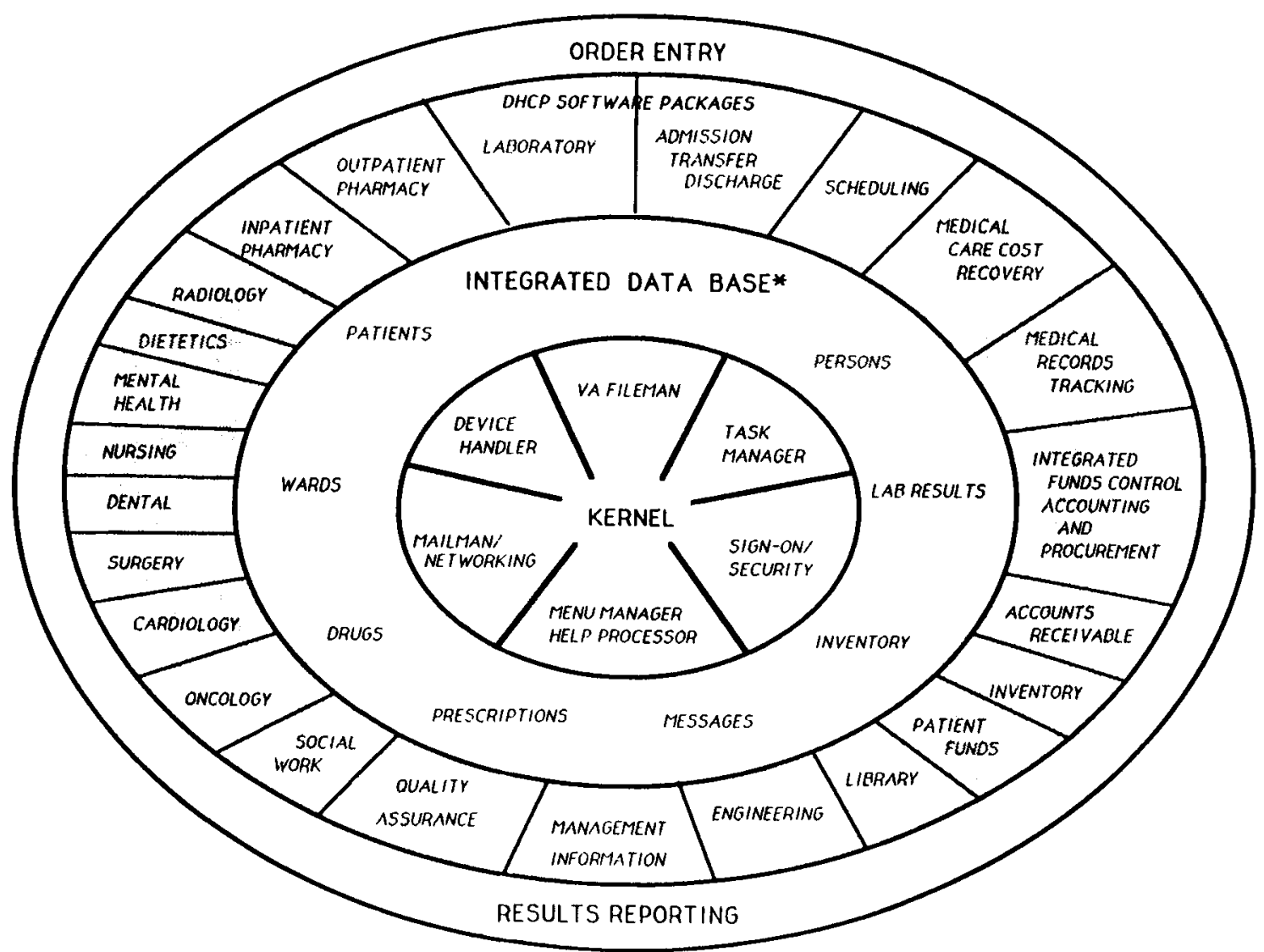

Fig 4. The VA's Overall DHCP Sottware Design. "Examples of files used by DHCP software packages. (Reprinted with permission. ${ }^{14}$ ) 


\section{VA'S DHCP INTEGRATED IMAGING PROJECT}

The US Department of Veterans Affairs has taken the direct approach outlined above in the conceptual model and described in Fig 3 to provide images to its physicians. ${ }^{15-17}$ A distributed system that provides image management and communications functionality as an integral part of the existing integrated hospital information system has been developed and tested. The network provides connectivity for multiple image servers (both magnetic and optical), 80386based high resolution true color image workstations, and the networked minicomputer-based DHCP hospital information system. ${ }^{18}$ Installation of a demonstration hospital-wide network is now underway.

Several criteria were considered when establishing this workstation network architecture. The system has a modular design so that, as technology advances in a particular area, individual components can be upgraded without affecting the rest of the system. Text and image data must be accessed over a high-speed network from a variety of operating systems running on equipment from a number of different manufacturers. The data server(s) for patient text data and digital image files may or may not be located on the same computer system depending upon the system architecture. Digital images will be stored on multiple media types including both magnetic and optical. The workstation needs local disk drives to temporarily store image files and software during a work session in order to minimize network traffic. In general, the VA's DHCP development goals include the production of vendor-independent, transportable systems through adherence to standards.

\section{CONCLUSIONS}

The VA's project to provide IMACS functionality within a hospital information system is unique because it is attempting integration at a higher level in the hospital information hierarchy than has been done before, it is a multiway communication system, and it can handle a wide variety of medical images reaching beyond radiology. We believe all of these features are necessary to the long-term viability of IMAC. The VA undertook this project with an integrated hospital information system written by its own staff and a large installed base of these systems to share the benefits. Future plans include the inclusion of other data types such as EKG's and audio data within the integrated HIS. This unique development environment presents the opportunity to explore the implications of HIS/IMAC integration for the next generation of hospital information systems.

\section{REFERENCES}

1. Shannon, RH: IMACS and Radiology: Defining the Problems, First International Conference on Image Management and Communication, Washington DC, 1989

2. McCoy W: The Software Services Backplane, Presented at the VA Symposium on High-Speed Communications in Support of Images in Hospital Information Systems, Washington DC, 1989

3. Arenson RL, Seshadri SB, Kundel HL, et al: PACS at Penn, Proc Society of Photo-optical Instrumentation Engineers Medical Imaging III, 1989, pp 50-59

4. Levine B, Meissner M, Benson H, et al: Integration of a RIS with an IMACS. Proc Society of Photo-optical Instrumentation Engineers Medical Imaging III, 1989, pp 183-191

5. Lodder H, van Poppel BM, de Valk JP, et al: HISPACS Coupling in practice. Proc Society of Photo-optical Instrumentation Engineers Medical Imaging III, 1989, pp 301-306

6. Boehme J, Choplin RH, Maynard D: Early experience in interfacing PACS to RIS. Proc Society of Photo-optical Instrumentation Engineers Medical Imaging III, 1989, pp $589-595$
7. ACR-NEMA Digital Imaging and Communications Standard 300-1985, National Electrical Manufacturers Association Wash DC

8. Prior F, Nabijee KH: Information Management for Data Retrieval in a PACS. Proc Society of Photo-optical Instrumentation Engineers Medical Imaging III, 1989, pp 488-497

9. HL7 Application Protocol for Electronic Data Exchange in Health Care Environment (HL7), HL7 Working Group, c/o S Schultz II, Hospital of the University of Pennsylvania, Kuljian Bldg, 2nd Floor, 3700 Market St, Philadelphia, PA 19104

10. Institute of Electrical and Electronic Engineers P1157: Medical Data Interchange Standard (MEDIX), Jack Harrington, Chair, Hewlett Packard, 3000 Minuteman Rd, Andover, MA 01810

11. Dayhoff, RE: Multimedia databases in MUMPS: An object-oriented approach. MUG Quarterly 18(4):15-18, 1989

12. Brannigan V: Procurement of Hospital Information Systems in the Federal Republic of Germany, MUG Quarterly, 17(3):13-19, 1987-88

13. VA Fileman Used Worldwide, US Medicine, July 1990 
14. Decentralized Hospital Computer Program, Department of Veterans Affairs, Medical Information Resources Management Office, Washington, DC 20420, 1989

15. Dayhoff RE: A medical image database system using a write once optical disk. Proc 11th Annual Symposium on Computer Applications in Medical Care, Institute of Electrical and Electronic Engineers, Washington, DC, 1987, pp 549-552

16. Dayhoff, RE, Maloney DL, Shepard BM: Integration of Images with the Veterans Affairs Hospital Information System in a Distributed Environment, Proc of the Symposium on Computer Applications in Medical Care, Institute of Electrical and Electronic Engineers, 1989
17. Dayhoff RE, Maloney DL: Providing PACS Functionality as an Integral Part of an Existing Hospital Information System, Proc Society of Photo-optical Instrumentation Engineers Medical Imaging IV, 1990

18. von Blankensee HT: VA DHCP Replacement Systems: VAXclusters for the 90's. Proc of the 1989 MUMPS Users' Group Meeting, MUG Quarterly 19(1):80-81, 1989

19. Dayhoff RE, Maloney DL, Kuzmak PM, et al: Demonstration of Dept of VA's Hospital Wide Image Workstation Network System, Proc of the Symposium on Computer Applications in Medical Care, Institute of Electrical Engineers, 1990 